\title{
Study of nuclear media effects in meson photoproduction
}

\author{
A. Lapik ${ }^{1}, V . \operatorname{Lisin}^{1}, V$. Nedorezov ${ }^{1, *}, A$. Polonski ${ }^{1}$, and N. Rudnev ${ }^{1}$ \\ for the BGO-OD collaboration \\ ${ }^{1}$ INR RAS, Moscow, Russia
}

\begin{abstract}
In addition to the current BGO-OD experimental program we propose to study nuclear media effects in photoproduction of mesons using a new experimental method based on measuring a recoil nucleon as a tagger of the selected partial channel. The principal goal is to learn how unstable mesons $\left(\pi^{o}, \pi^{+}, \pi^{-}, \eta, \rho, \omega\right.$, etc. $)$ interact with nuclear media. This approach continues previous GRAAL experiments to study nuclear excitation dynamics, including multifragmentation of nuclei by intermediate energy photons.
\end{abstract}

\section{Introduction}

Current BGO-OD experiments are devoted mainly to strangeness photoproduction at intermediate energies [1]. This experiment at the ELSA accelerator facility uses the energy tagged bremsstrahlung photon beam to investigate the excitation spectrum of the nucleon. The setup (see Fig. 1) consists of a highly segmented BGO calorimeter surrounding the target, with a particle tracking magnetic spectrometer at forward angles. BGO-OD is ideal for investigating the photoproduction of hadrons of non-zero strangeness. The high momentum resolution at forward angles covers the kinematic region.

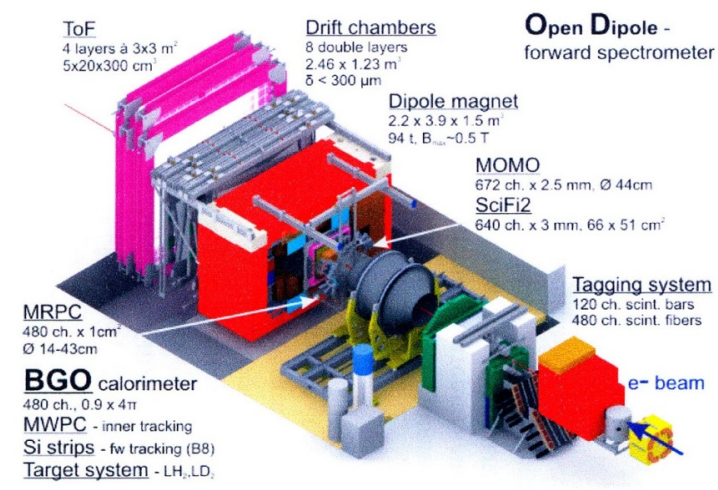

Figure 1. Set-up of the BGO-OD experiment

\footnotetext{
*e-mail: vladimir@cpc.inr.ac.ru
} 
Preliminary BGO-OD experimental results [1] (see Fig. 2) demonstrate a high momentum resolution of the recoil nucleons from kaon meson production. A principal improvement as compared with GRAAL has been obtained due to the magnetic spectrometer placed downstream of the target to distinguish charged mesons, protons, deuterons and other charged products. So, the BGO-OD facility is appropriate to study the nuclear excitation dynamics using the recoils as taggers.
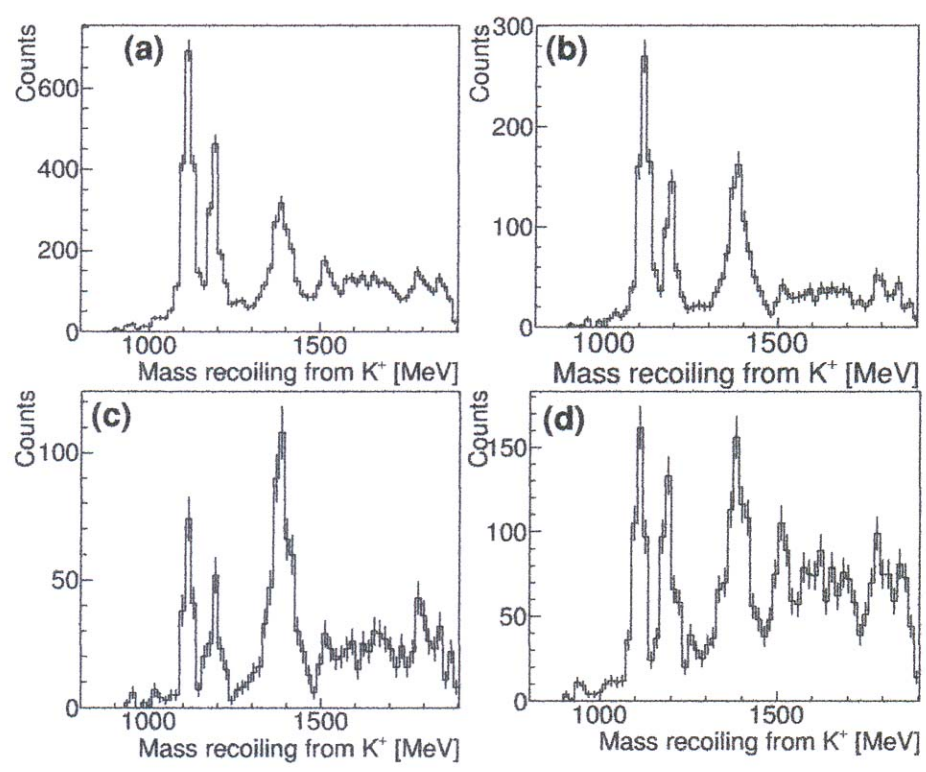

Figure 2. Preliminary results on mass recoiling in kaon photoproduction off the proton [1]

\section{Description of the new method and experimental requirements}

Simulations performed with the GEANT-4 code illustrate the idea presented firstly in [2]. Fig. 3 shows the simulated recoil proton energy distributions vs the photon energy for $14 \mathrm{~N}$ nuclei. One can see that recoil proton variables indicate clearly the type of the reaction. It would be stressed here that no kinematics analysis (either invariant or missing mass) is used here. Only these physical properties of the detector are required which make it possible to carry out measurement and separation of protons with high resolution as it can be evaluated from Fig. 3.

Therefore, some developments to improve the detector parameters have been done. First of all, we studied the proton detection efficiency in the Barrel, i.e. the ratio of events when the proton was detected on the targets. It is seen in Fig. 4 that the distributions are noticeably different for the measurements with the proton and carbon targets and do not depend on the photon energy regions. The difference between the proton detection efficiencies for the proton and carbon targets can be explained by the difference obtained due to the kinematical restrictions for event selections at different targets. This analysis is important to study the meson photoproduction on the nuclei with high multiplicity. 


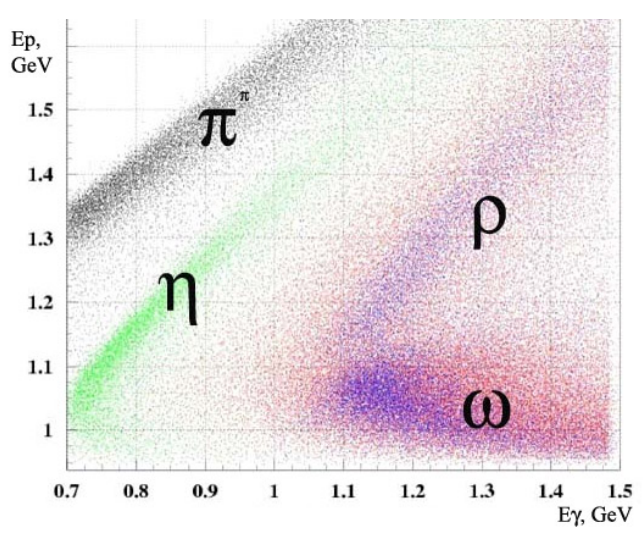

Figure 3. Energy of recoil proton vs the $E_{\gamma}$ energy. Angle $\theta_{p}$ is limited: $2^{\circ}<\theta_{p}<10^{\circ}$
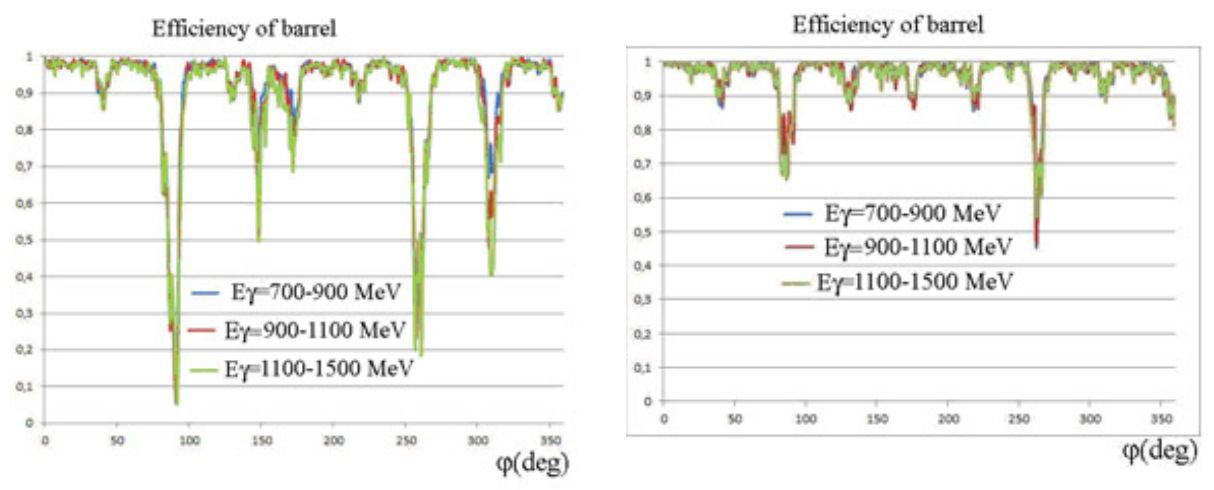

Figure 4. Proton detection efficiency in Barrel for proton (left) and ${ }^{12} \mathrm{C}$ target

The principal problem to realize this method is to distinguish the primary and secondary recoils which can be produced in the intranuclear cascade process initiated by the elementary meson photoproduction. The multifragmentation process in light nuclei has been studied recently in detail [3]. Fortunately, the probability of cascade interaction for primary nucleon does not exceed $20 \%$, at least, in light nuclei, the probability of multifragmentation is small. Additionally we can use the latest data showing that the energy and angular distributions of primary and secondary nucleons are quite different. Fig. 5 shows the GRAAL experimental results on the energy and angular distributions of recoil protons; all partial channels for $E_{\gamma}=$ $800-1500 \mathrm{MeV}$ have been included. One can see that the primary recoil proton which has the maximal energy is emitted in the forward direction while cascade protons have isotropic distributions.

Separation of charged particles is based on the TOF method in the forward direction and $\Delta E$ measurement at large solid angles, obtained earlier in the GRAAL experiment, is shown in Fig. 6 [3].

One can see that the experimental resolution allows one to separate protons from pions but not to distinguish protons and light fragments either in the forward or BGO direction. So, the OD magnet in the forward direction is very suitable to improve the resolution by 10 times 

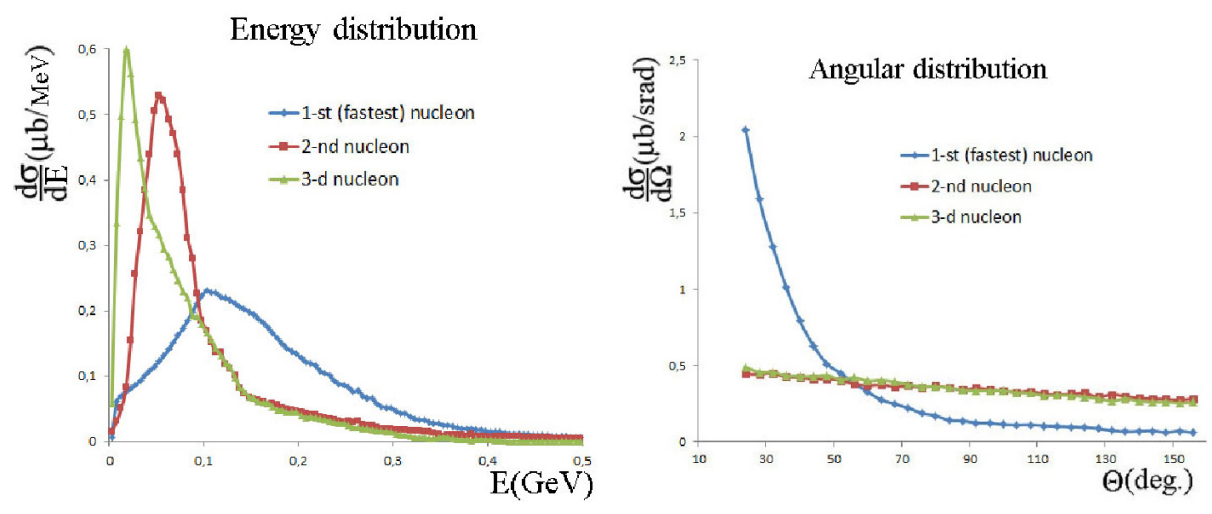

Figure 5. Energy and angular distributions of the recoil protons
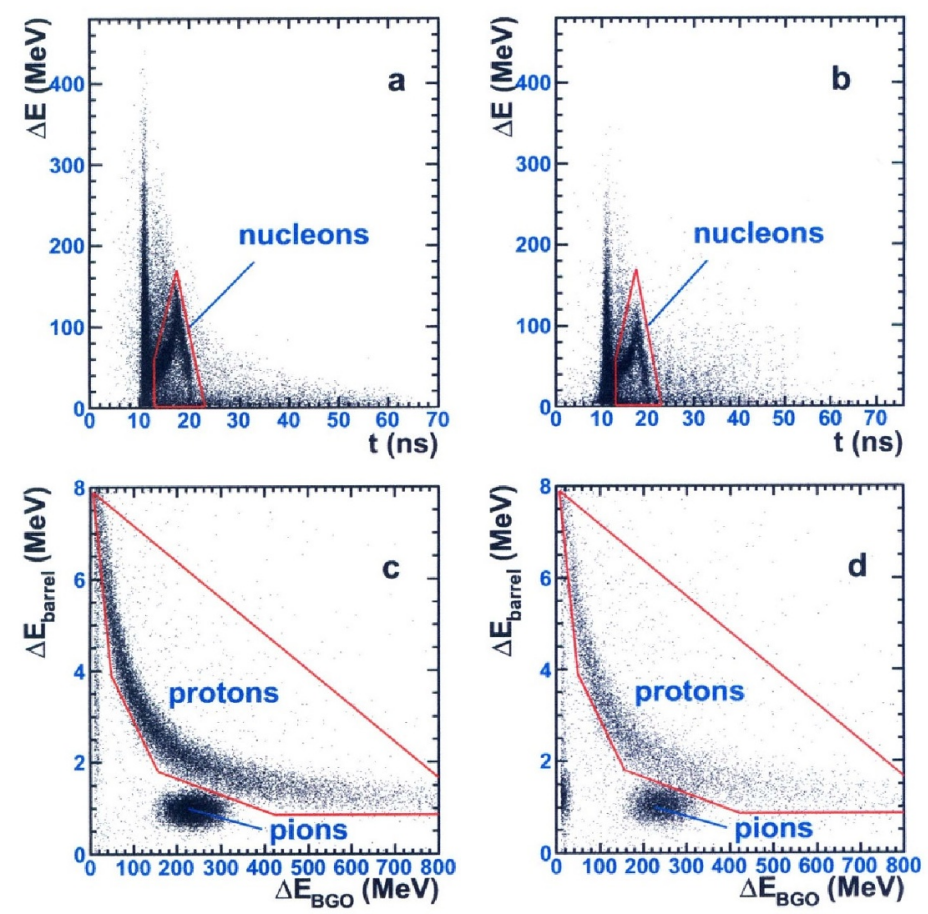

Figure 6. GRAAL results for the forward detector ( $(\Delta E$ - TOF) and BGO ball $\Delta E$ (barrel) - $\Delta E(\mathrm{BGO})$

at least. Also, it would be reasonable to include the analysis of the analog $\Delta E$ signals from MWPC to separate light fragments (deuterons, alphas etc) from protons, as shown in Fig. 7.

\section{World results available for comparison}

The existing approaches to study the interactions of unstable mesons with nuclear media are scarce. We can mention the recent experiment to study inelastic interaction of $\eta^{\prime}$ mesons with 


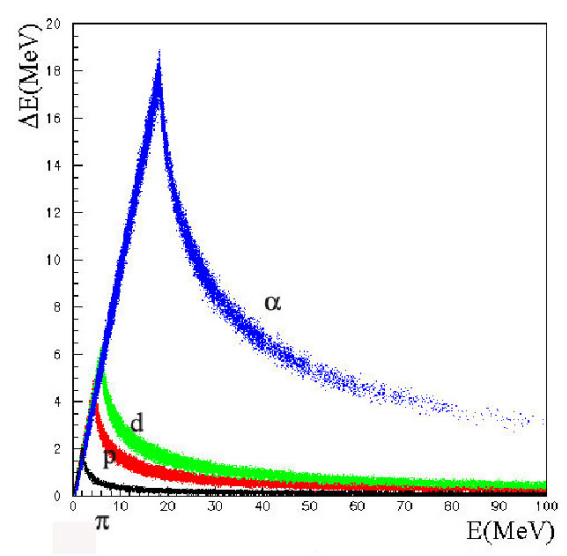

Figure 7. Energy losses of protons and light fragments in MWPC according to the simulation

nuclear media [4] which was based on measuring the transparency factor $T_{A}$ evaluated in the Glauber model with the eikonal approximation:

$$
\tilde{T}_{A}=\frac{\sigma_{\gamma A \rightarrow \eta^{\prime} A^{\prime}}}{A \sigma_{\gamma N \rightarrow \eta^{\prime} N}}
$$

Here $\sigma$ is the partial cross section, A - the atomic number.

Normalized to ${ }^{12} \mathrm{C}$ nucleus:

$$
T_{A}=\frac{\pi R^{2}}{A \sigma_{\eta^{\prime} N}}\left\{1+\left(\frac{\lambda}{R}\right) \exp \left[-2 \frac{R}{\lambda}\right]+\frac{1}{2}\left(\frac{\lambda}{R}\right)^{2}\left(\exp \left[-2 \frac{R}{\lambda}\right]-1\right)\right\} .
$$

The evaluated inelastic cross section for $\eta^{\prime}$ mesons was found to be equal to $10.3 \pm 1.4 \mathrm{mb}$ for $E_{\gamma}=1.5-2.2 \mathrm{GeV}, \theta_{p}=1^{o}-11^{o}$. This result can be used in future for comparison.

\section{Conclusion}

The future BGO-OD experiment using the recoil nucleon is a tagger of the partial meson photoproduction interesting in different aspects of research in dependence of multiplicity on recoil nucleons. The multiplicity equals to one corresponds to elastic scattering reactions induced by unstable mesons and the recoil nucleon is emitted dominantly in the forward direction. For multiplicity $n=2$ we have the inelastic interaction of unstable meson with nuclear media. For example, the reaction $\eta n \rightarrow \pi^{-} p$ can be studied for comparison with existing data [4]. The multiplicity $n>2$ corresponds to the multifragmentation of nucleus which can be considered as a phase transition between the nuclear matter and gas of nucleons and fragments. The most interesting case corresponds to the multiplicity $n=0$ which indicates the coherent interaction including Delbruck scattering [5], low energy and momentum transfer photofission reactions [6] etc. It would be stressed that the photon beam is necessary for this kind of experiments because a nucleus is transparent for photons (universal curve), background reactions (elastic and multiple scattering of projectiles ) are negligible, multiplicity of products is relatively small to identify them completely. So, the BGO-OD experiments allow one to solve the fundamental problems mentioned above. At the first stage it is recommended to use the ${ }^{12} \mathrm{C}$ target for calibration by comparing the data with the GRAAL data [3]. 


\section{References}

[1] T. Jude et al. (BGO-OD collaboration), PoS Hadron 2017, 054 (2018). doi:10.22323/1.310.0054

[2] A. Ignatov et al., Prog.Part.Nucl.Phys. 61, 253-259 (2008)

[3] V. Nedorezov et al. (GRAAL collaboration), Nuclear Physics A 940, 264-278 (2015)

[4] M. Nanova et al. (BGO-OD collaboration), Phys. Lett. B 710, 600-606 (2012)

[5] Heishun Zen, Masaki Fujimoto, Takehito Hayakawa, Masahiro Katoh, Toshiteru Kii, James Koga, Hideaki Ohgaki, Elham Salehi, Toshiyuki Shizuma, J. Phys.: Conf. Ser. 1067(9), 092003 (2018). doi:10.1088/1742-6596/1067/9/092003

[6] G.Ya. Kezerashvili, V.V. Muratov, V.G. Nedorezov, A.S.Sudov, Rus. J. Nucl. Phys. 55(1), 3 (1992) 